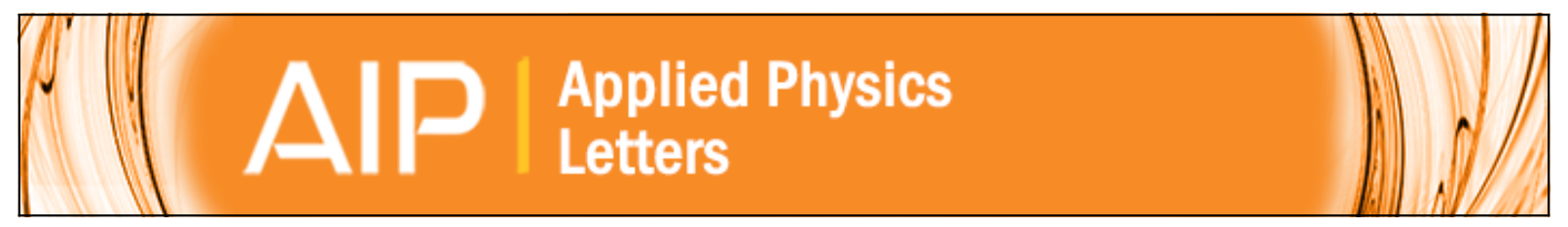

\title{
Near-field spectroscopy of phase segregation in white-light-emitting blends based on low-mass molecules
}

Marco Salerno, Marco Mazzeo, Maria C. Frassanito, Salvatore Patanè, Roberto Cingolani, and Giuseppe Gigli

Citation: Applied Physics Letters 86, 081907 (2005); doi: 10.1063/1.1867556

View online: http://dx.doi.org/10.1063/1.1867556

View Table of Contents: http://scitation.aip.org/content/aip/journal/apl/86/8?ver=pdfcov

Published by the AIP Publishing

\section{Articles you may be interested in}

Tunable blue organic light emitting diode based on aluminum calixarene supramolecular complex

Appl. Phys. Lett. 85, 10 (2004); 10.1063/1.1767274

Point Light Sources Based on Color Centers in LiF Films for Scanning NearField Optical Microscopy

AIP Conf. Proc. 709, 444 (2004); 10.1063/1.1764050

Monolayer topography resolution achieved in a scanning near-field optical microscope

Rev. Sci. Instrum. 73, 4250 (2002); 10.1063/1.1519936

Near-field photoluminescence spectroscopy of InGaN films grown by molecular-beam epitaxy

Appl. Phys. Lett. 80, 989 (2002); 10.1063/1.1446206

Ultraviolet-visible near-field microscopy of phase-separated blends of polyfluorene-based conjugated semiconductors

Appl. Phys. Lett. 79, 833 (2001); 10.1063/1.1389822

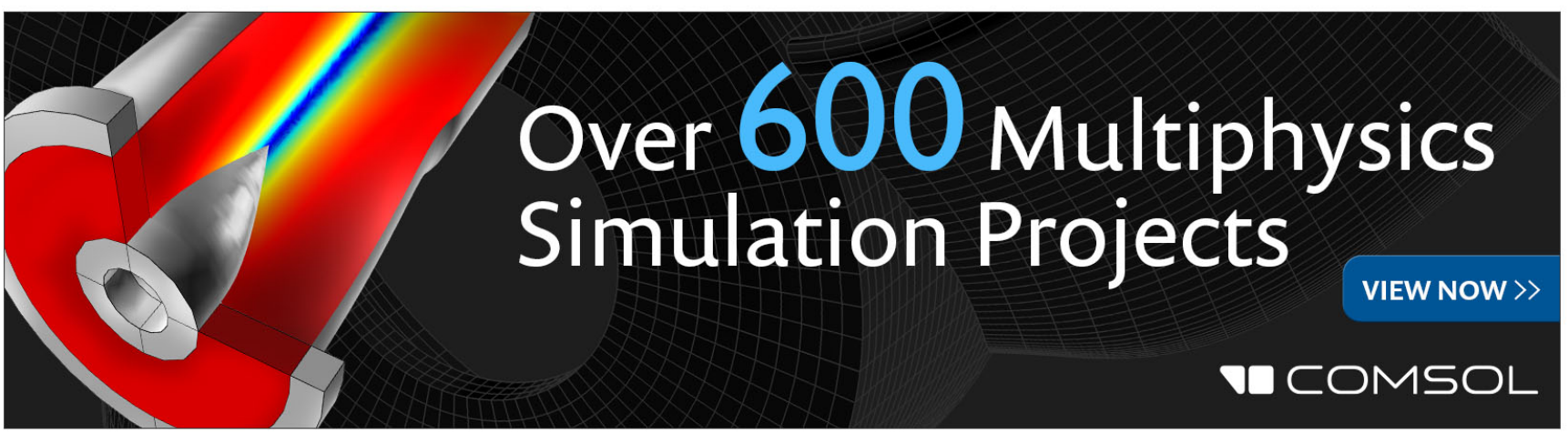




\title{
Near-field spectroscopy of phase segregation in white-light-emitting blends based on low-mass molecules
}

\author{
Marco Salerno, ${ }^{\text {a) }}$ Marco Mazzeo, and Maria C. Frassanito \\ National Nanotechnology Laboratory of INFM, via Arnesano km 5, I-73100 Lecce, Italy \\ Salvatore Patanè \\ INFM and Dipartimento Fisica della Materia e Tecnologie Fisiche Avanzate, Università di Messina, salita \\ Sperone 31, I-98166 Messina, Italy \\ Roberto Cingolani and Giuseppe Gigli \\ National Nanotechnology Laboratory of INFM, via Arnesano km 5, I-73100 Lecce, Italy
}

(Received 5 August 2004; accepted 22 December 2004; published online 16 February 2005)

\begin{abstract}
We report on the direct observation of phase segregation occurring in thin-film blends of a thiophene monomer and an ammino compound, used in the fabrication of organic white-light-emitting diodes. In the homogeneous and uniform regions of the films, the interaction between the two molecular components gives rise to exciplex states responsible for a broad redshifted photoluminescence emission band, which disappears in the film zones where segregation occurs. This effect has been observed with submicrometer spatial resolution by means of local spectroscopic measurements performed in a scanning near-field optical microscope. () 2005 American Institute of Physics. [DOI: $10.1063 / 1.1867556]$
\end{abstract}

Over the last years, an increasing effort has been devoted to the development of white-light-emitting diodes based on luminescent organic compounds (WOLEDs). ${ }^{1-3}$ These materials are quite promising for the fabrication of large-area and high efficiency devices, thus being attractive for illumination applications, such as backlighting and headlighting. A common approach to obtain white light from WOLEDs relies on the superposition of emission at different wavelengths originated from independent active materials. ${ }^{4,5}$ An alternative route is based on the electron recombination from intermolecular levels formed in a molecular blend, often referred to as the bulk exciplex approach. ${ }^{6,7}$ Compared to multistep sequential evaporation or coevaporation of low-mass active molecules, 8,9 the exciplex approach requires a simpler deposition process, such as spin casting of a solution containing the different molecules into a single active layer, and the resulting electroluminescence is found to be independent of the applied voltage. ${ }^{10}$ In this case, the uniformity of the active layer is a key issue for the optical properties of the device. In fact, the morphology of fine grained materials following deposition is characterized by labile equilibrium states. ${ }^{11}$ This is especially true for thin films where critical boundary conditions apply, and in polymer films where nucleation/coalescence and spinodal decomposition are commonly observed upon sample aging, even in the presence of constant environmental parameters. ${ }^{12-14}$ In this frame, a deep understanding of the correlation between optical and morphological properties in a molecular film device is of crucial importance to achieve full control of the device characteristics.

Atomic force microscopy (AFM) is a well-known and valuable tool to investigate the morphology of thin films. On the other hand, surface mapping of the optical properties at nanometer scale is only possible by means of scanning nearfield optical microscopy (SNOM). In this letter, we apply

\footnotetext{
${ }^{a)}$ Electronic mail: marco.salerno@unile.it
}

these two techniques to investigate the relationship between optical and morphological properties in a WOLED based on the bulk exciplex approach. In particular, we study a binary blend of two blue-emitting molecules, namely, 2,5bis(trimethylsilyl)-thiophene-1,1-dioxide (STO) (Refs. 15,16) and N,N'-diphenyl-N,N'-bis(3-methylphenyl)-1,18biphenyl-4,4'diamine (TPD) (Ref. 17) whose bulk exciplex emission results in a white-light spectrum. SNOMphotoluminescence (PL) spectra have been correlated to the occurrence of clusterization in the spin-coated films. The measured local suppression of PL clearly demonstrates a phase segregation effect observed in low molecular mass compounds.

The STO and TPD molecules have already been demonstrated to form exciplex when blended together, and give rise to bright white emission in the solid state. ${ }^{18,19}$ Their molecular structure and the electronic levels involved in exciplex formation are displayed in Fig. 1. The STO/TPD blend films investigated in this work are prepared by dissolving a 1:1 mass ratio of the two molecules in toluene $(4.0 \mathrm{mg}$ of each in

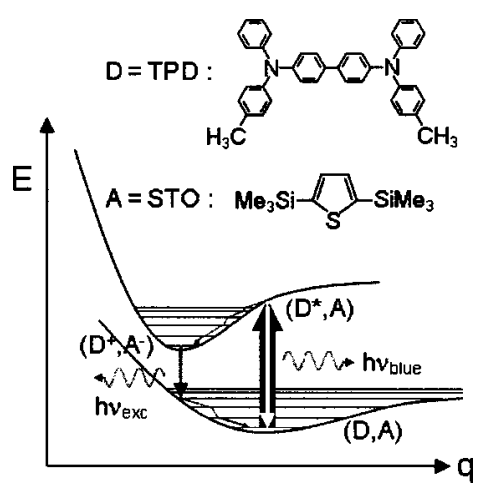

FIG. 1. Molecular structures and energetic levels of the STO/TPD blend, showing the single molecule highest occupied molecular orbital-lowest unoccupied molecular orbital $\left(h \nu_{\text {blue }}\right)$ and the exciplex transition $\left(h \nu_{\text {exc }}\right)$. D: Electron donor, A: Electron acceptor. 


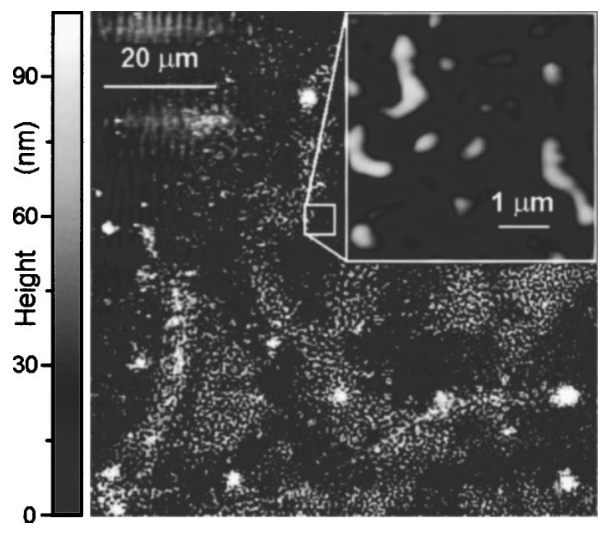

FIG. 2. AFM topography of a STO/TPD blend film, (scan size 90 $\times 90 \mu \mathrm{m}^{2}$, relative height range see color bar). Inset: Zoom on the 5 $\times 5 \mu \mathrm{m}^{2}$ region enclosed in the white square.

$0.59 \mathrm{ml}$ solvent), and spin casting the solution onto quartz glass substrates, at a speed of $2000 \mathrm{rpm}$ for $1 \mathrm{~min}$. Solvent evaporation is allowed to occur at room temperature. The relative composition of the blends expressed in terms of STO molar concentration over the molar concentration of the overall dissolved solute (STO+TPD) is $66.5 \%$.

The AFM observation of our samples has been carried out by means of a commercial NTMDT AFM operating in tapping mode. A surface morphology characterized by a nonuniform distribution of clusters and ripples has been observed in all the measured films (see Fig. 2). These structures are supposed to grow up as a consequence of phase segregation of the STO/TPD blend, due to a critical balance of relative component concentration. ${ }^{18}$ Therefore, the higher features should be either STO rich or TPD rich, as opposed to the continuous and comparatively flat film regions surrounding them. In order to verify this hypothesis, we used a homemade SNOM setup to spatially resolve the emission of our samples. The SNOM was equipped with a nonoptical shear force detection mechanism ${ }^{19}$ using a $33 \mathrm{kHz}$ tuning fork on which a UV commercial probe (Nanonics) was glued, with a $50 \mathrm{~nm}$ nominal aperture size. Surface scanning was always performed in illumination-reflection mode, namely, illuminating the sample through the aperture and collecting the reflected light in the far field. A He-Cd laser line at $325 \mathrm{~nm}$ wavelength and a Hamamatsu R-943 photomultiplier tube were used as the light source and the detector, respectively. The optical data collected refer to the luminescence signal alone, as we used standard (non-UV) optical fiber and objective to couple the far field to the phototube, which cut off the elastically scattered laser light.

To avoid photobleaching due to repeated scanning of the same areas, a topographical mapping without optical imaging was first performed, in order to identify relevant protruded structures in the flat surrounding film region. At this point, we switched on the SNOM mode and repeated the scan to locally collect the PL. Representative topography and optical map are shown in Fig. 3. The topographical features are consistent with those imaged by AFM (see inset of Fig. 2). The optical map clearly shows a lower light intensity from the protrusion clusters, due to the disappearance of the exciplex band in the green-red region of the spectrum. This is demonstrated in the spatially resolved mapping of the SNOM-PL spectra taken at selected positions on the probed sample area. A line scan across the imaged cluster [gray hori-

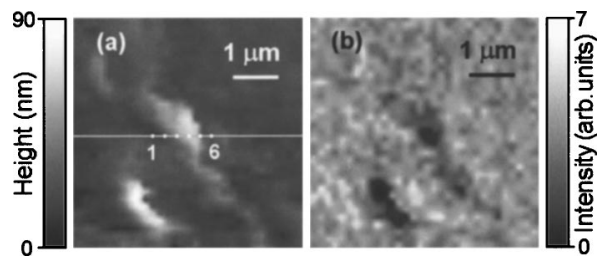

FIG. 3. (a) Constant shear-force topography image, (sample area 5 $\times 5 \mu \mathrm{m}^{2}, 64 \times 64$ pixel map). (b) SNOM image, collected in reflection simultaneously to (a).

zontal line in Fig. 3(a)] was performed at a conveniently low speed (220 s per line) to allow for a good optical signal detection (i.e., high signal-to-noise ratio). The reflection SNOM signal was sent to a Jobin-Yvon charge coupled device camera equipped with a Triax 320 monochromator as a detector. Spectra were acquired along the SNOM scan line with an integration time of $3.5 \mathrm{~s}$ each, corresponding to a spatial resolution of $\sim 250 \mathrm{~nm}$. Figure 4 reports the normalized PL spectra collected at the positions identified in Fig. 3 (a) by white spots along the gray line (numbered 1 to 6 from left to right). All of the spectra show a peak at $420 \mathrm{~nm}$ wavelength, which is in good agreement with the expected PL from the individual molecules. Points 1 and 6, right outside the cluster, show the broad exciplex emission in the blend around $570 \mathrm{~nm}$. This low-energy band is usually observed in the topographically flat regions of the film, as all other points of the investigated line, with minor changes in profile and peak intensity (data not shown). When the SNOM probe tip crosses the cluster, on the contrary, a strong reduction of the exciplex emission is observed, roughly proportional to the distance from the cluster boundary (Points 2-5), with a minimum of intensity occurring at the central region (Point 4). The decrease of the exciplex emission was repeatedly observed in all clusters and indicates the occurrence of local quenching of the exciplex due to segregation of the blend in the protruded region.

The spectra of Fig. 4 have to be compared with the integrated PL spectra of similar samples reported in a previous work. ${ }^{18}$ In that case, a lower STO concentration (53\%) was compatible with the presence of the exciplex band, whereas a concentration similar to the present one (67\%) showed no exciplex band over all samples. ${ }^{20}$ Concurrently, the appearance of morphological features, such as clusters and ribbons, in the film suggested the occurrence of phase segregation in the blend. Such a hypothesis is here demonstrated by the SNOM-PL measurements, which allowed us to distinguish

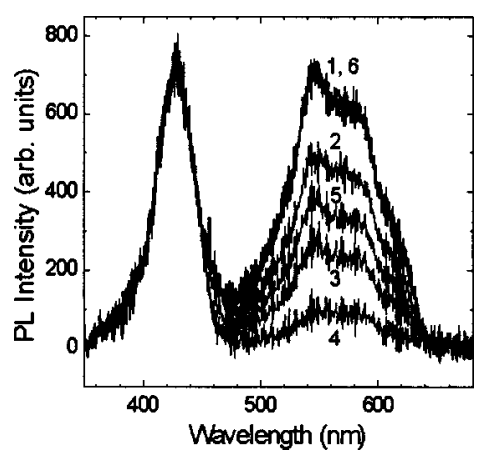

FIG. 4. SNOM-PL spectra collected at Positions 1-6 along the line scan through the cluster in (a) (gray line). Intensities have been normalized to the peak on the left side. 
the different optical behavior of submicrometer spaced regions of the sample surface. The presence of a more structured exciplex band, as compared to Ref. 18, can be due to the activation of local exciplexes of different conformation, i.e., different emission energy ${ }^{10}{ }^{10}$ which are spatially resolved here by the SNOM-PL as opposed to standard macro-PL characterization.

In conclusion, we performed near-field optical spectroscopy on a binary blend of a thiophene-based oligomer and a diamine derivative. These molecules are of great technological interest in the field of WOLEDs. By focusing the attention on the correlation between morphological and optical properties of clusters formed in the blend, we were able to show phase segregation in such low molecular mass materials. Observation and control of this phenomenon can be quite important to forecast the performance limits of WOLED devices with active layers based on similar molecules and improve their design.

${ }^{1}$ R. S. Deshpande, V. Bulović, and S. R. Forrest, Appl. Phys. Lett. 75, 888 (1999).

${ }^{2}$ C. W. Ko and Y. T. Tao, Appl. Phys. Lett. 79, 4234 (2001).

${ }^{3}$ K. O. Cheon and J. Shinar, Appl. Phys. Lett. 84, 1201 (2004).

${ }^{4}$ L. Zugang and H. Nazare, Synth. Met. 111, 47 (2000).

${ }^{5}$ Z. Xie, Y. Li, J. Huang, Y. Wang, C. Li, and J. Shen, Synth. Met. 106, 71
(1999).

${ }^{6}$ The Exciplex, edited by M. Gordon and W. R. Ware (Academic, New York, 1975).

${ }^{7}$ C.-I. Chao and S.-A. Chen, Appl. Phys. Lett. 73, 426 (1998).

${ }^{8}$ C.-H. Kim and J. Shinar, Appl. Phys. Lett. 80, 2201 (2002).

${ }^{9}$ Y.-S. Huang, J.-H. Jou, W.-K. Weng, and J.-M. Liu, Appl. Phys. Lett. 80, 2782 (2002).

${ }^{10}$ M. Mazzeo, D. Pisignano, F. Della Sala, J. Thompson, R. I. R. Blyth, G. Gigli, R. Cingolani, G. Sotgiu, and G. Barbarella, Appl. Phys. Lett. 82, 334 (2003).

${ }^{11}$ H. Ramanarayan and T. A. Abinandanan, Bull. Mater. Sci. 26, 189 (2003).

${ }^{12}$ H. Ade, D. A. Winesett, A. P. Smith, S. Qu, S. Ge, J. Sokolov, and M. Rafailovich, Europhys. Lett. 45, 526 (1999).

${ }^{13}$ A. Bernasik, J. Włodarczyk-Miśkiewicz, W. Łużny, K. Kowalski, J. Raczkowska, J. Ryszc, and A. Budkowski, Synth. Met. 144, 253 (2004).

${ }^{14}$ S. Minko, M. Müller, D. Usov, A. Scholl, C. Froeck, and M. Stamm, Phys. Rev. Lett. 88, 035502 (2002).

${ }^{15}$ G. Barbarella, O. Pudova, C. Arbizzani, M. Mastragostino, and A. Bongini, J. Org. Chem. 63, 1742 (1998).

${ }^{16}$ G. Barbarella, L. Favaretto, G. Sotgiu, M. Zambianchi, L. Antolini, E. A. Marseglia, E. Tedesco, G. Gigli, and R. Cingolani, Synth. Met. 115, 47 (2000).

${ }^{17}$ J. Thompson, R. I. R. Blyth, M. Mazzeo, M. Anni, G. Gigli, and R. Cingolani, Appl. Phys. Lett. 79, 560 (2001).

${ }^{18}$ M. Mazzeo, J. Thompson, R. I. R. Blyth, M. Anni, G. Gigli, and R. Cingolani, Physica E (Amsterdam) 13, 1243 (2002).

${ }^{19}$ K. Karrai and R. Grober, Appl. Phys. Lett. 66, 1842 (1995).

${ }^{20} \mathrm{~A}$ systematic investigation of the correlation between STO concentration and phase segregation is the subject of a forthcoming paper. 